\title{
Epidemiologia e organização de serviços de saúde: diabetes mellitus numa comunidade de Porto Alegre
}

\author{
Epidemiology and organization of health services: \\ diabetes mellitus in a community of the city of Porto Alegre
}

${ }^{1}$ Programa de PósGraduação em Saúde Coletiva, Universidade do Vale do Rio dos Sinos. Av. Unisinos 950, S. João Batista. 93022-000 São Leopoldo RS. episoares@terra.com.br ${ }^{2}$ Departamento de Medicina Social, Faculdade de Medicina, Universidade Federal de Pelotas
Abstract This study sought to assess the conditions of processing and results in care for people aged over 20 diagnosed with diabetes mellitus and living in an area covered by the UBS Vila Gaúcha, in Porto Alegre. Disease control rate of interviewees with self-reported diabetes was assessed. Definition of this rate was established from arterial pressure values, glycosylated hemoglobin, abdominal circumference, and/or BMI. Of the 753 people aged over 20 years, 37 (4.9\%; 95\% CI 3.4 6.5) mentioned diabetes mellitus diagnosed by a physician. Disease control rate measurements were performed in 31 (83.8\%) people. Results revealed $24(77.4 \%)$ individuals with altered blood pressure levels, 25 (80.6\%) with BMI equal to or above $25 \mathrm{~kg} / \mathrm{m}^{2}, 29$ (93.5\%) with abdominal circumference above normal and 18 (58.1\%) with glycosylated hemoglobin equal to or above $8 \%$. Diagrams were used to analyze disease control rate factors. Only one patient was classified as controlled. Diagram interpretation points to the need for policies and actions aiming to reduce body weight in the community, which would include practically all patients with diabetes.

Key words Diabetes mellitus, Quality of healthcare, Primary healthcare, Cross-Sectional studies, Process and outcome assessment
Resumo O objetivo foi avaliar as condições de processo e de resultado no atendimento às pessoas acima de 20 anos com diagnóstico de diabetes mellitus, residentes na área de cobertura da UBS Vila Gaúcha, Porto Alegre. Avaliou-se a prevalência de pacientes controlados com diabetes mellitus. A definição de pacientes controlados foi estabelecida a partir dos valores da pressão arterial, hemoglobina glicada, circunferência abdominal e/ou IMC. Das 753 pessoas acima de 20 anos, 37 (4,9\%; IC95\% 3,4-6,5) referiram diabetes mellitus diagnosticado por médico. Realizaram-se as medidas de controle em 31 (83,8\%) pessoas. Encontraram-se 24 $(77,4 \%)$ indivíduos com níveis pressóricos alterados, 25 (80,6\%) com IMC igual ou maior que 25 $\mathrm{kg} / \mathrm{m}^{2}, 29(93,5 \%)$ com circunferência abdominal acima da normalidade e 18 (58,1\%) com hemoglobina glicada igual ou maior que $8 \%$. Para se avaliar os resultados foram utilizados diagramas. Apenas um paciente foi classificado como controlado. A interpretação dos diagramas aponta para a necessidade de ações visando à redução de peso corporal na comunidade, medida que atingiria os pacientes com diabetes.

Palavras chave Diabetes mellitus, Qualidade dos cuidados de saúde, Atenção primária à saúde, Estudos transversais, Avaliação de processos e resultados 


\section{Introdução}

O Brasil está passando por uma transição demográfica devido ao declínio das taxas de fertilidade e de mortalidade, assim, tem aumentado a proporção de indivíduos com 50 anos ou mais de idade. Com o envelhecimento da população, as doenças não transmissíveis têm se tornado mais frequentes e provocam mudanças na organização do sistema de saúde ${ }^{1,2}$.

Atualmente, as doenças não transmissíveis são responsáveis por $66 \%$ da carga de doenças no Brasil, caracterizando-se pelos anos de vida perdidos por morte prematura e por incapacitação ${ }^{1}$. Entre as doenças crônicas não transmissíveis o diabetes mellitus se destaca pela sua morbimortalidade e como fator de risco para patologias do aparelho circulatório. No Brasil, estima-se que entre 2000 e 2030 o número de indivíduos com diabetes passará de 4,5 milhões para 11 milhões $^{3}$, sendo que a metade desta população desconhecerá o diagnóstico ${ }^{2}$.

A avaliação da qualidade da atenção básica é importante para a escolha de prioridades, para a determinação de metas das ações de saúde e para a organização dos serviços de saúde ${ }^{4}$.

O diagnóstico da situação de saúde possibilita estimativa de frequência de doenças e das características dos cuidados oferecidos pelos serviços, podendo fornecer informações importantes para a organização das ações na rede de atenção básica. Neste sentido, torna-se necessária a incorporação do método epidemiológico no auxílio à gestão, pois permite avaliar com precisão para que se possa escolher e implementar ações que apresentem uma maior efetividade, menores efeitos colaterais e a melhor relação custo-benefício ${ }^{2,5-7}$.

O presente estudo avaliou as condições de processo e de resultado no cuidado a esses pacientes portadores de diabetes.

\section{Métodos}

Foi realizado um inquérito epidemiológico incluindo toda a população residente na Vila Gaúcha. O trabalho de campo foi produzido entre fevereiro e maio de 2008. Os objetivos deste inquérito foram: estabelecer a cobertura de procedimentos preventivos na população residente e organizar as atividades da Unidade Básica de Saúde.

A segunda etapa, que compreendeu o presente estudo, foi realizada a partir da identificação no inquérito dos indivíduos com 20 anos ou mais de idade, de ambos os sexos, que referiram diagnós- tico médico de diabetes mellitus e que não estavam hospitalizados no momento da entrevista.

Entre as 37 pessoas identificadas com diabetes mellitus, em $31(83,8 \%)$ foi possível realizar as medidas de hemoglobina glicada, os níveis pressóricos e de antropometria. Foram perdidas as informações de seis pessoas (duas mudaram de endereço, duas se recusaram a coletar medidas e duas foram hospitalizadas).

Foram aplicados questionários padronizados e pré-codificados. As entrevistas foram realizadas por entrevistadores submetidos a treinamentos de aplicação e padronização de medidas ${ }^{8}$. O controle de qualidade foi realizado através de reaplicação de instrumento simplificado em 5\% da população incluída no estudo.

A dimensão que expressou resultado foi a prevalência de pacientes controlados com diabetes mellitus referido. A definição de prevalência de pacientes controlados foi estabelecida a partir dos valores da pressão arterial, de hemoglobina glicada e circunferência abdominal e, ou, índice de massa corporal (IMC) $)^{9,10}$.

Para a medida da pressão arterial foram utilizados esfigmomanômetros analógicos da marca Becton e Dickinson modelo Premium, devidamente aferidos e calibrados pelo INMETRO. As medidas de pressão arterial foram coletadas segundo preconizado pela Pan American Hypertension Initiative ${ }^{11}$. Assim, foram realizadas as médias das duas últimas medidas das pressões sistólicas e diastólicas. Valores iguais ou superiores a 140/90 $\mathrm{mm} \mathrm{Hg}$ foram considerados alterados ${ }^{12}$.

Os indivíduos foram pesados com o menor número de roupas possíveis, descalços, em balança mecânica com régua antropométrica de 192 $\mathrm{cm}$, da marca Filizola, modelo 31, com capacidade máxima para $150 \mathrm{Kg}$. As medidas de altura foram aferidas mantendo os entrevistados com os ombros levantados, pescoço reto, olhando fixamente para frente. As balanças foram calibradas semanalmente com peso padrão de $1 \mathrm{Kg}$. O índice de massa corporal foi calculado pela razão do peso, em quilos, multiplicado pela altura, em metros ao quadrado. Foi considerado como aceitável o IMC abaixo de $25 \mathrm{Kg} / \mathrm{m}^{2}$, entre homens e mulheres, conforme os critérios da American Diabetes Association ${ }^{10}$.

A circunferência da cintura foi medida através do perímetro abdominal, em centímetros, medido no local de máxima extensão da região do abdômen ${ }^{13}$, utilizando-se uma fita milimetrada (Graphco), com $180 \mathrm{~cm}$ de comprimento, flexível, não elástica, colocada diretamente sobre a pele no momento da expiração. Os indivíduos, no momento 
deste procedimento, estavam em pé, com o abdômen desnudo. O ponto de corte para obesidade abdominal foi igual ou superior a $80 \mathrm{~cm}$ para mulheres e igual ou superior a $94 \mathrm{~cm}$ em homens $\mathrm{s}^{14}$.

Sabe-se que os resultados da hemoglobina glicada refletem a glicemia média dentro de dois ou três meses precedentes ${ }^{9,10,15}$. A Hemoglobina Glicada foi medida da seguinte maneira: primeiramente o laboratório de análises clínicas do Hospital Mãe de Deus disponibilizou as coletas de sangue e as análises dos exames. Após, os indivíduos da amostra foram contatados pelo supervisor de campo que agendou horários no laboratório. Os testes foram realizados com base no método laboratorial de cromotografia líquida de alta eficiência (CLAE) que mede exatamente o conteúdo de hemoglobina glicada. Foram consideradas como normais medidas de até $8 \% \%^{9,10,15}$.

Para a construção de indicadores de processo, que expressaram as características da assistência, foram coletadas informações sobre atendimento médico no último ano e no último mês que precederam a entrevista, que incluiu o número, o local de consultas e se recebia tratamento do mesmo profissional. Também foi investigado se as pessoas receberam orientação de qualquer profissional de saúde sobre cuidados com os pés, de dieta e de atividade física. Verificou-se também se os pacientes realizavam dieta, atividade física e uso de medicamentos.

Para a descrição da população foram coletadas informações demográficas, socioeconômicas, das características da doença e do seu manejo.

Quanto às características da doença foi verificado o tempo de diagnóstico, a história familiar de diabetes (pai e mãe) e as hospitalizações alguma vez na vida pela doença. Estimou-se a ocorrência de possíveis complicações microangiopáticas e macroangiopáticas. Como representação de algumas complicações microangiopáticas indagou-se se algum médico disse que o entrevistado tinha problemas nos rins e ou nos olhos causados por diabetes mellitus. Além disso, foi investigado se algum médico tinha referido diabetes mellitus como causa de infarto e de derrame, representando algumas das possíveis complicações macroangiopáticas.

Os dados tiveram dupla digitação, para a diminuição de erros de consistência, através do software Epi-Info v.6.

A análise bruta dos dados foi realizada no software SPSS v.16, com a descrição das características da amostra, da doença e do manejo.

Foram construídos diagramas para se verificar a distribuição e as combinações dos critérios da prevalência de pacientes controlados: o primeiro incluiu hemoglobina glicada, pressão arterial e circunferência abdominal; o segundo foi constituído por hemoglobina glicada, pressão arterial e índice de massa corporal.

O projeto foi aprovado pelo Comitê de Ética e Pesquisa do Hospital Mãe de Deus.

\section{Resultados}

Das 753 pessoas entrevistadas com 20 ou mais anos de idade, 37 (4,9\%; IC95\% 3,4 - 6,5) referiram diabetes mellitus diagnosticado por médico.

A amostra foi predominantemente composta por pessoas do sexo feminino $(59,5 \%)$, com idade média de 53 anos, não brancas (59,5\%), em união estável $(54,1 \%)$ e com escolaridade até o ensino fundamental incompleto $(83,8 \%)$. Verificou-se que $35,1 \%$ dos entrevistados ainda fumavam e somente um homem apresentou excesso de uso habitual de bebidas alcoólicas.

Em relação às características da doença, pouco mais da metade da amostra $(51,4 \%)$ tinha três anos ou menos como tempo de diagnóstico inicial, sendo que a média foi de seis anos. Verificou-se também que $24,3 \%$ dos indivíduos tinham história familiar positiva e $16,2 \%$ das pessoas hospitalizaram alguma vez na vida por causa de diabetes. Quanto à ocorrência de manifestações referidas por médico e causadas por diabetes mellitus, 48,6\% referiram problemas nos olhos, $32,4 \%$ infarto e ou derrame, e 13,5\% problemas nos rins (Tabela 1).

A análise mostrou que $13(35,1 \%)$ pessoas não referiram nenhuma alteração devido à doença.

Os resultados mostraram que $24(77,4 \%)$ indivíduos apresentavam níveis pressóricos alterados, $25(80,6 \%)$ com IMC igual ou maior que $25 \mathrm{~kg} / \mathrm{m}^{2}, 29(93,5 \%)$ com valores de circunferência abdominal acima dos níveis de normalidade e 18 (58,1\%) com níveis de hemoglobina glicada igual ou maior que $8 \%$ (Tabela 2). Em relação às combinações dos critérios de prevalência de pacientes controlados, foi constatado que apenas um paciente estava com a doença controlada (Figura 1, Figura 2).

Ao se incluir medida de circunferência abdominal na Figura 1, observaram-se dois $(6,4 \%)$ indivíduos com um critério isolado de não controle. No segundo Diagrama, substituindo-se a circunferência abdominal pelo índice de massa corporal, constataram-se cinco $(16,1 \%)$ entrevistados com critérios isolados de não controle (Figura 2). 
Tabela 1. Distribuição das características dos pacientes com diabetes mellitus. Vila Gaúcha, Porto Alegre, RS, 2008. ( $\mathrm{N}=37)$

\begin{tabular}{lrr}
\hline & N & $\%$ \\
\hline Tempo de diagnóstico & & \\
$\quad 4$ anos ou mais & 18 & 48,6 \\
$\quad$ anos ou menos & 19 & 51,4 \\
História familiar de diabetes & & \\
$\quad$ Não & 28 & 75,7 \\
$\quad$ Um dos genitores & 6 & 16,2 \\
$\quad$ Ambos os genitores & 3 & 8,1 \\
Hospitalização na vida por causa & & \\
do diabetes & & \\
$\quad$ Não & 31 & 83,8 \\
$\quad$ Sim & 6 & 16,2 \\
Problemas nos olhos por causa & & \\
do diabetes & & \\
$\quad$ Não & 19 & 51,4 \\
$\quad$ Sim & 18 & 48,6 \\
Problemas nos rins por causa do & & \\
diabetes & & \\
$\quad$ Não & 32 & 86,5 \\
Sim & 5 & 13,5 \\
Problemas nos rins e/ou nos \\
olhos por causa do diabetes
\end{tabular}

Tabela 2. Distribuição de algumas características de condições físicas da amostra. Vila Gaúcha, 2008. $(\mathrm{N}=31)$

\begin{tabular}{lrr}
\hline & N & $\%$ \\
\hline Pressão Arterial & & \\
$\quad$ Compensada & 7 & 22,6 \\
$\quad$ Não compensada & 24 & 77,4 \\
IMC & & \\
$\quad<25 \mathrm{Kg} / \mathrm{m}^{2}$ & 6 & 19,4 \\
$\quad \geq 25 \mathrm{Kg} / \mathrm{m}^{2}$ & 25 & 80,6 \\
Circunferência abdominal & & \\
$\quad$ Normal & 2 & 6,5 \\
$\quad$ Acima do normal & 29 & 93,5 \\
Hemoglobina Glicada & & \\
$\quad<8 \%$ & 13 & 41,9 \\
$\quad \geq 8 \%$ & 18 & 58,1 \\
\hline
\end{tabular}

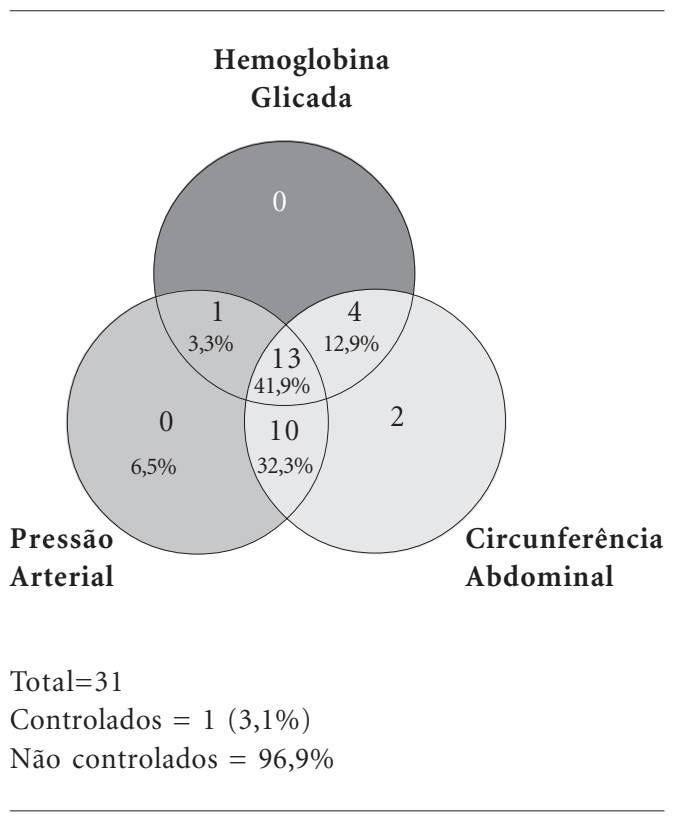

Figura 1. Combinações dos critérios de prevalência de controlados. Vila Gaúcha, Porto Alegre, RS, 2008. $(\mathrm{N}=31)$

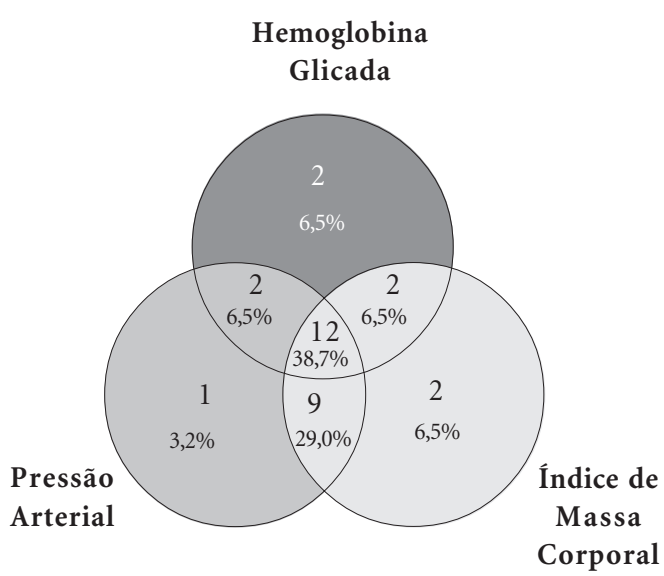

Total $=31$

Controlados $=1(3,1 \%)$

Não controlados $=96,9 \%$

Figura 2. Combinações dos critérios de prevalência de controlados. Vila Gaúcha, Porto Alegre, RS, 2008. $(\mathrm{N}=31)$ 
Em relação aos indicadores de processo, verificou-se que $64,9 \%$ dos indivíduos consultaram com médico pela doença no último ano que precedeu a entrevista, sendo que a média foi de três consultas por entrevistado. Entre as 14 $(37,8 \%)$ pessoas que consultaram por diabetes mellitus no último mês antes da entrevista, 70,8\% referiram como local de consulta a Unidade Básica de Saúde Vila Gaúcha. Entre todos os entrevistados com a doença $62,2 \%$ consultavam sempre com o mesmo médico. A maior parte dos pacientes recebeu orientação de algum profissional sobre dieta e atividade física, $30(81,1 \%)$ e 24 $(64,9 \%)$, respectivamente, entretanto $64,9 \%$ não foram informados sobre os cuidados com os pés. (Tabela 3).

Quanto ao manejo, 40,5\% dos entrevistados referiram realizar dieta e $24,3 \%$ faziam atividade física e quase a metade, $45,9 \%$ não utilizavam medicamentos (Tabela 4).

Tabela 3. Distribuição das características de assistência aos pacientes com diabetes. Vila Gaúcha, Porto Alegre, RS, 2008. (N=37)

\begin{tabular}{lrr}
\hline \multicolumn{1}{l}{ N } & $\%$ \\
\hline $\begin{array}{l}\text { Consultou com médico no } \\
\text { último ano? }\end{array}$ & & \\
$\quad$ Sim & 24 & 64,9 \\
$\quad$ Não & 13 & 35,1 \\
Consultou com médico no & & \\
último mês? & & \\
$\quad$ Sim & 14 & 37,8 \\
$\quad$ Não & 23 & 62,2 \\
Onde consultou a última vez? & & \\
$\quad$ U.B.S. Vila Gaúcha & 17 & 70,8 \\
$\quad$ Hospital & 5 & 20,8 \\
$\quad$ Outros postos & 2 & 8,3 \\
Consulta sempre com o & & \\
mesmo médico? & & \\
$\quad$ Sim & 23 & 62,2 \\
$\quad$ Não & 14 & 37,8 \\
Recebeu aconselhamento sobre & & \\
dieta & & \\
$\quad$ Sim & 30 & 81,1 \\
$\quad$ Não & 7 & 18,9 \\
Recebeu aconselhamento sobre \\
atividade física
\end{tabular}

\section{Discussão}

Diagnósticos comunitários de saúde são importantes para a compreensão das características de doenças em populações específicas, exigem tempo reduzido para coleta de dados e análise, além de apresentarem baixo custo ${ }^{16}$.

A prevalência de diabetes mellitus encontrada foi de 4,9\% (IC95\% 3,4-6,5) e está de acordo com alguns estudos de base populacional realizados no Brasil. Theme-Filha et al. ${ }^{17}$ mostraram prevalência de 6,2\% (IC95\% 5,4-7,0) de pessoas com diabetes na Pesquisa de Saúde Mundial realizada no país em $2003^{17}$, a Pesquisa Nacional de Amostra de Domicílio encontrou 4,3\% na Região Sul ${ }^{18} \mathrm{e}$ o estudo transversal realizado por Dias da Costa et al. ${ }^{19}$ na cidade de Pelotas demonstrou frequência de 5,6\% (IC95\% 4,6-6,6) da doença.

Para a organização de serviços de saúde devese destacar que do total de indivíduos com diabetes apenas metade é conhecedor da enfermidade, assim, a carga da doença deve ser maior do que a encontrada ${ }^{20}$. Vale ressaltar que a população incluída no estudo vive em área delimitada fisicamente e de grande vulnerabilidade social, demonstrada pela baixa escolaridade. Portanto são necessários outros estudos que tenham como objetivo o rastreamento e a captação de pacientes não diagnosticados, para a melhor compreensão das características da doença nesta população.

Quanto aos indicadores de resultado do manejo da doença, utilizaram-se diagramas para análise dos fatores de controle. Na construção dos diagramas apenas um paciente foi classificado como controlado durante o estudo. Assim,

Tabela 4. Distribuição das características de manejo dos pacientes com diabetes. Vila Gaúcha, Porto Alegre, RS, 2008. $(\mathrm{N}=37)$

\begin{tabular}{lrr}
\hline & N & $\%$ \\
\hline Está fazendo dieta? & & \\
$\quad$ Sim & 15 & 40,5 \\
$\quad$ Não & 22 & 59,5 \\
Está fazendo atividade física? & & \\
$\quad$ Sim & 9 & 24,3 \\
$\quad$ Não & 28 & 75,7 \\
Uso de medicamentos & & \\
$\quad$ Não utiliza medicamentos & 17 & 45,9 \\
Glibenclamida e Metformina & 8 & 21,6 \\
Glibenclamida & 4 & 10,8 \\
$\quad$ Metformina & 4 & 10,8 \\
Clorpropamida & 1 & 2,7 \\
Insulina NPH & 3 & 8,1 \\
\hline
\end{tabular}


os resultados confirmaram toda a complexidade do manejo da doença. O UKPDS mostrou que aproximadamente $50 \%$ dos pacientes foram incapazes de manter níveis $<7 \%$ de hemoglobina glicada por três anos e $75 \%$ não o fizeram em nove anos ${ }^{21}$. Sabe-se que nos Estados Unidos menos do que $12 \%$ dos pacientes diagnosticados alcançam as metas preconizadas para os níveis de glicemia, colesterol sanguíneo e pressão arterial $^{22}$. No Sul do Brasil, em estudo transversal avaliando o manejo de pacientes com diabetes mellitus na rede pública, foram encontrados entre $6 \%$ e $11 \%$ de pacientes controlados ${ }^{6}$.

A elevada proporção de pacientes que não controlam a doença pode contribuir para o aumento das complicações cardiovasculares, determinando, assim, um prognóstico desfavorável. Da mesma forma, as complicações estão relacionadas diretamente a um aumento da mortalidade dos indivíduos com diabetes ${ }^{23-26}$.

$\mathrm{Na}$ análise da Figura 1, apenas um indivíduo classificado como não controlado não mostrou envolvimento do aumento da cintura abdominal entre as combinações. Enquanto que na Figura 2, cinco pessoas não apresentavam excesso de peso. Portanto, o uso do critério antropométrico cintura abdominal mostrou maior sensibilidade para a classificação de controle, mais uma vez mostrando a circunferência da cintura como a medida antropométrica melhor correlacionada à quantidade de tecido adiposo visceral, reconhecido fator de risco para doenças cardiovasculares, diabetes, dislipidemias e síndrome metabólica ${ }^{27}$.

Atualmente, as intervenções em relação ao diabetes mellitus preconizam manejo de risco multifatorial ${ }^{28,29}$. Entretanto, a interpretação dos diagramas aponta para a necessidade de políticas e ações visando à redução de peso corporal na comunidade, como medidas que atingiriam praticamente todos os pacientes com diabetes, beneficiando também aqueles com pré-diabetes, que não foram incluídos no estudo.

Quanto às características da amostra, foram identificados $83,8 \%$ das pessoas com até o nível fundamental escolar incompleto, estando de acordo com outros estudos que também apontaram prevalências maiores de diabetes em indivíduos com menor nível socioeconômico ${ }^{6,17,30}$. Evidentemente que os níveis de descontrole da doença e de baixas condições socioeconômicas podem explicar o elevado percentual de indivíduos referindo manifestações secundárias tanto microangiopáticas como macroangiopáticas. A amostra apresentou um percentual de $32,4 \%$ dos indivíduos com manifestações no aparelho circulatório por causa de diabetes: infarto e derrame cerebral, determinando um pior prognóstico. Entretanto, conforme Braga et al. ${ }^{26}$, as intervenções neste grupo trazem maior impacto quanto aos benefícios, tanto a curto quanto em longo prazo ${ }^{26}$.

Em relação à assistência a saúde, $64,9 \%$ dos sujeitos com diabetes mellitus consultaram no último ano, enquanto que $37,8 \%$ consultaram no último mês. Em relação à última consulta, 70,8\% dos diabéticos consultaram na Unidade Básica de Saúde Vila Gaúcha, e 62,2\% consultaram sempre com o mesmo médico. Esperava-se um percentual maior de pacientes referindo o uso de serviços de saúde. Em estudo de base populacional realizado no sul do Brasil, na cidade de Pelotas, envolvendo 3100 adultos com mais de 20 anos, constatou-se que houve associação positiva de diabetes com a maior probabilidade de consulta com médico ${ }^{31}$. Outro estudo realizado na mesma cidade, na população de 20 a 69 anos, apontou que todos os indivíduos que apresentavam doenças crônicas, incluindo diabetes mellitus, quase dobraram a probabilidade de consultarem mais de oito vezes ao ano comparados aos indivíduos sem essas condições ${ }^{32}$. Assim, os achados do presente estudo apontaram a necessidade de se fazer busca ativa dos pacientes com diabetes mellitus intensificando os cuidados necessários.

Os dados mostraram diferenças importantes quanto às recomendações sobre a doença, $81,1 \%$ receberam aconselhamento sobre dieta, $64,9 \%$ sobre atividade física e $35,1 \%$ sobre cuidados com os pés. Deve-se ressaltar que não existe uma hierarquia que faça com que alguma dessas recomendações seja mais prescrita do que outra, todas consideradas essenciais para o cuidado da doença ${ }^{33,34}$.

Quanto ao tratamento, identificou-se baixa adesão dos pacientes às mudanças de estilo de vida, $75,7 \%$ não realizavam atividade física e 59,5\% não faziam dieta. Reconhece-se que as modificações relacionadas aos hábitos de vida são lentas, demandam tempo e esforço dos profissionais de saúde. Entretanto ações educativas devem ser reforçadas, nos moldes da continuidade, persistência e comunicação adequada, para que o conhecimento gere mudança de vida para os pacientes com diabetes. Os resultados mostraram uma proporção de sedentarismo alta, pois $75,7 \%$ dos entrevistados afirmaram não estar fazendo atividade física regularmente, embora mais da metade da amostra, 64,9\%, tenha recebido alguma orientação profissional sobre este item. Portanto, apenas informação não é suficiente para a alteração de hábitos de vida. Ainda sobre o tratamento, 
outro dado chamou atenção, 45,9\%, quase metade das pessoas com a doença, referiu não utilizar nenhum medicamento, hoje disponíveis na rede básica através do Plano de Reorganização da Atenção à Hipertensão Arterial ${ }^{7}$.

A contribuição da atenção básica para a promoção da equidade tem se destacado, principalmente nas sociedades com diferenças sociais ${ }^{4}$. As populações menos favorecidas são as que mais necessitam de políticas públicas que objetivem o melhoramento das suas condições de vida, cri-

\section{Colaboradores}

RG Mielczarski: coleta de dados, supervisão do trabalho de campo, análise dos dados e redação do artigo. JSD Costa e MTA Olinto: elaboração do projeto, análise dos dados e redação do artigo. ando cenários que estabeleçam novos paradigmas de saúde. As dificuldades para realizar práticas de saúde que estejam de acordo com os conhecimentos científicos estabelecidos e suas correlações com as condições inadequadas devem ser compreendidas para serem modificadas.

Modificações de algumas condições independem da equipe de saúde, como por exemplo, saneamento básico e renda familiar, assim tornamse importantes serem estudados aqueles fatores que podem mais facilmente ser modificados.

\section{Referências}

1. Banco Mundial. Unidade de Gerenciamento do Brasil. Unidade de Gestão do Setor de Desenvolvimento Humano. Região da América Latina e do Caribe. Enfrentando o Desafio das Doenças Não Transmissíveis no Brasil. Documento do Banco Mundial. Relatório No 32576; 2005. [acessado 2008 set 29]. Disponível em: http://www-wds.worldbank.org/external/ default/WDSContentServer/WDSP/IB/2005/12/30/ 000160016_20051230115919/Rendered/PDF/325760 PORTUGUESE0BR0Gray0Cover.pdf

2. Paiva DCP, Bersusa APS e Escuder MML. Avaliação da assistência ao paciente com diabetes e/ou hipertensão pelo Programa Saúde da Família do Município de Francisco Morato, São Paulo, Brasil. Cad Saude Publica 2006; 22(2):377-385.

3. Wild S, Roglic G, Green AA, Sicree R, King H. Global Prevalence of Diabetes Estimates for the year 2000 and projections for 2030. Diabetes Care 2004; 27(5):1047-1053.

4. Szwarcwald CL, Mendonça MHM, Andrade CLT. Indicadores de atenção básica em quatro municípios do Estado do Rio de Janeiro, 2005: resultados de inquérito domiciliar de base populacional. Cien Saude Colet 2006; 11(3):643-655.

5. César CLG, Tanaka OY. Inquérito domiciliar como instrumento de avaliação de serviços de saúde: um estudo de caso na região sudoeste da área metropolitana de São Paulo, 1989-1990. Cad Saude Publica 1996; 12(Supl. 2):S59-S70.

6. Assunção MCF, Santos IS, Gigante DP. Atenção primária em diabetes no Sul do Brasil: estrutura, processo e resultado. Rev Saude Publica 2001; 35(1):88-95.

7. Brasil. Ministério da Saúde (MS). Secretaria de Políticas Públicas. Plano de Reorganização da Atenção à Hipertensão Arterial e ao Diabetes Mellitus: fase de detecção de casos suspeitos de DM. Rev Saude Publica 2001; 35(5):490-493. 
8. Brasil. Ministério da Saúde (MS). Secretaria de Políticas Públicas. Departamento de Atenção Básica. Saúde da Criança: acompanhamento do crescimento e desenvolvimento infantil. Padronização controle de qualidade de medidas antropométricas. Brasília: Ministério da Saúde (MS); 2002.

9. Sociedade Brasileira de Diabetes (SBC). Diretrizes da SBD sobre o tratamento e acompanhamento do Diabetes Mellitus. Rio de Janeiro: Diagraphic Editora; 2006.

10. American Diabetes Association. Standards of Medical Care in Diabetes-2007. Diabetes Care 2007; 30(Suppl. 1):S4-S41.

11. Pan American Hypertension Initiative. Working meeting on blood pressure measurement: sugges tions for measuring blood pressure to use in populations surveys. Rev Panam Salud Publica 2003 14(5):300-302.

12. The Seventh Report of the Joint National Committee on Prevention, Detection, Evaluation and Treatment of High Blood Pressure - The JCN 7 Report. JAMA 2003; 289(19):2560-2572.

13. Lohman TG, Roche AF, Martorell R. Anthropomet ric Standardization Reference Manual. Champaign: Human Kinetics Books; 1988.

14. Olinto MTA, Nacul LC, Dias da Costa JS, Gigante DP, Menezes AMB, Macedo S. Níveis de intervenção para obesidade abdominal: prevalência e fatores associados. Cad Saude Publica 2006; 22(6):12071215 .

15. Grupo Interdisciplinar de Padronização da Hemoglobina Glicada - Alc. A importância da hemoglobina glicada (A1c) para a avaliação do controle glicêmico em pacientes com diabetes mellitus: aspectos clínicos e laboratoriais. 2004; (1). [acessado 2008 set 29]. Disponível em: http://www.diabetes.org.br/educacao/ docs/hemoglic2004.pdf

16. Viacava F. Informações em saúde: a importância dos inquéritos populacionais. Cien Saude Colet 2002; 7(4):607-621

17. Theme-Filha MM, Szwarcwald CL, Souza-Júnior PRB. Socio-demographic characteristics, treatment coverage and self-rated health individuals who reported six chronic diseases in Brazil, 2003. Cad Saude Publica 2005; 21(Supl. 1):S43-S53.

18. Barros MBAB, César CLG, Carandina L, Torre GD. Desigualdades sociais na prevalência de doenças crônicas no Brasil. PNAD-2003. Cien Saude Colet 2006; 11(4):911-926.

19. Costa JSD, Olinto MTA, Assunção MCF, Gigante DP, Macedo S, Menezes AMB. Prevalência de Diabetes Mellitus em Pelotas, RS: um estudo de base populacional. Rev Saude Publica 2006; 40(3):542-545.

20. Malerbi DA, Franco LJ. Multicenter study of the prevalence of diabetes mellitus and impaired glucose tolerance in the urban Brazilian population aged 30-69 years. Diabetes Care 1992; 15(11):1509-1516.

21. Turner RC, Cull CA, Frighi V, Holman RR. UK Prospective Diabetes Study (UKPDS) Group. Glycemic control with diet, sulfonylurea, metformin, or insulin in patients with type 2 diabetes mellitus: progressive requirement for multiple therapies (UKPDS 49). JAMA 1999; 281(21):2005-2012.
22. Bruton SA, Rolla AR. Implementing intensified treatment strategies for patients with type 2 diabetes mellitus. J Fam Pract 2007; 56(11):S9-S16.

23. Melo MS, Lolio CA, Lucena MAF, Kirzner CF, Martins SM, Barros MNDS. Multiple causes of death in diabetic individuals in the Brazilian Northeastern Region. Rev Saude Publica 1991; 25(6):435-442.

24. Rodrigues TC, Pecis M, Azevedo MJ, Gross JL. Blood pressure homeostasis and microvascular complications in diabetic patients. Arq Bras Endocrinol Metabol 2005; 49(6):882-890.

25. Picon PX, Zanatta, CM, Gerchman F, Zelmanovitz T, Gross JL, Canani LH. Análise dos critérios de definição da síndrome metabólica em pacientes com diabetes melito tipo 2. Arq Bras Endocrinol Metabol 2006; 50(2): 264-270.

26. Braga JR, Santos ISO, Flato UP, Guimarães HP, Ave zum A. Impacto do diabetes mellitus na mortalidade em síndromes coronarianas agudas. Arq Bras Endocrinol Metabol 2007; 51(2):275-280.

27. Jansen I, Katzmarzyk PT, Ross R. Body mass index, waist circumference, and health risk. Arch Intern Med 2002; 162(18):2074-2079.

28. Gaede P, Vedel P, Larsen N, Jensen GVH, Parving $\mathrm{HH}$, Pedersen O. Multifactorial Intervention and Cardiovascular Disease in Patients with Type 2 Diabetes. New Engl J Med 2003; 348(5):383-393

29. Goff DC Jr, Gerstein HC, Ginsberg HN, Cushman WC, Margolis KL, Byington RP, Buse JB, Genuth S, Probstfield JL, Simons-Morton DG; ACCORD Study Group. Prevention of Cardiovascular Disease in Persons with Type 2 Diabetes Mellitus: Current Knowledge and Rationale for the Action to Control Cardiovascular Risk in Diabetes (ACCORD) Trial. Am J Cardiol 2007; 99(12A):4i-20i.

30. Araújo RB, Santos I, Cavaleti MA, Dias da Costa IS, Béria JU. Avaliação do cuidado prestado a pacientes diabéticos em nível primário. Rev Saude Publica 1999; 33(1):24-32

31. Capilheira MF, Santos IS. Fatores individuais associados à utilização de consultas médicas por adul tos. Rev Saude Publica 2006; 40(3):436-443.

32. Dias da Costa JS, Olinto MTA, Gigante DP, Menezes AMB, Macedo S, Daltoé T, Santos IS, Fuchs SC. Utilização de serviços ambulatoriais de saúde em Pelotas, Rio Grande do Sul, Brasil: alguns fatores relacionados com as consultas médicas acima da média. Cad Saude Publica 2008; 24(2):353-363.

33. Triplitt CL. New Technologies and Therapies in the Management of Diabetes. Am J Manag Care 2007; 13(Suppl. 2):S47-S54.

34. Hainer V, Toplak H, Mitrakou A. Treatment modalities of obesity: what fits whom? Diabetes Care 2008; 31(Supl. 2): S269-S277.

Artigo apresentado em 25/10/2009

Aprovado em 21/02/2010

Versão final apresentada em 15/03/2010 